Journal of the Society for the Study of Architecture in Canada Le Journal de la Société pour l'étude de l'architecture au Canada

\title{
Patrimoine et développement local
}

\section{Le cas de l'édifice RCA}

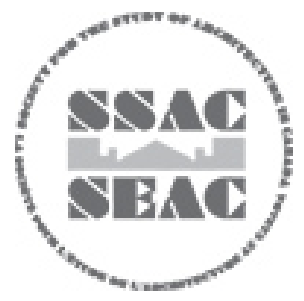

\section{Laurent Dambre-Sauvage}

Volume 45, numéro 2, 2020

Chercheurs en émergence

Emerging Scholars

URI : https://id.erudit.org/iderudit/1076488ar

DOI : https://doi.org/10.7202/1076488ar

Aller au sommaire du numéro

Éditeur(s)

SSAC-SEAC

ISSN

2563-8696 (numérique)

Découvrir la revue

Citer cet article

Dambre-Sauvage, L. (2020). Patrimoine et développement local : le cas de l'édifice RCA. Journal of the Society for the Study of Architecture in Canada / Le Journal de la Société pour l'étude de l'architecture au Canada, 45(2), 60-74. https://doi.org/10.7202/1076488ar d'utilisation que vous pouvez consulter en ligne. 


\section{PATRIMOINE ET DÉVELOPPEMENT LOCAL}

Le cas de l'édifice RCA

Diplômé en histoire de l'art, en chant lyrique et en science politique, LAURENT DAMBRE-SAUVAGE a été commissaire de l'exposition Lille au XVII siècle : des archiducs au Roi-Soleil au palais des Beaux-Arts de Lille, puis chargé de la régie et de la conservation préventive des collections au musée de la Chartreuse de Douai. Par la suite, il a collaboré avec diverses institutions musicales avant d'entreprendre un doctorat en études urbaines. II s'intéresse aux communs culturels et à la mobilisation de la culture et du patrimoine comme moteurs de développement local. Sous la direction de Lucie K. Morisset, il collabore à une étude de l'Organisation de coopération et de développement économiques (OCDE) sur les impacts en termes de développement local des actions du Musée des beaux-arts de Montréal.

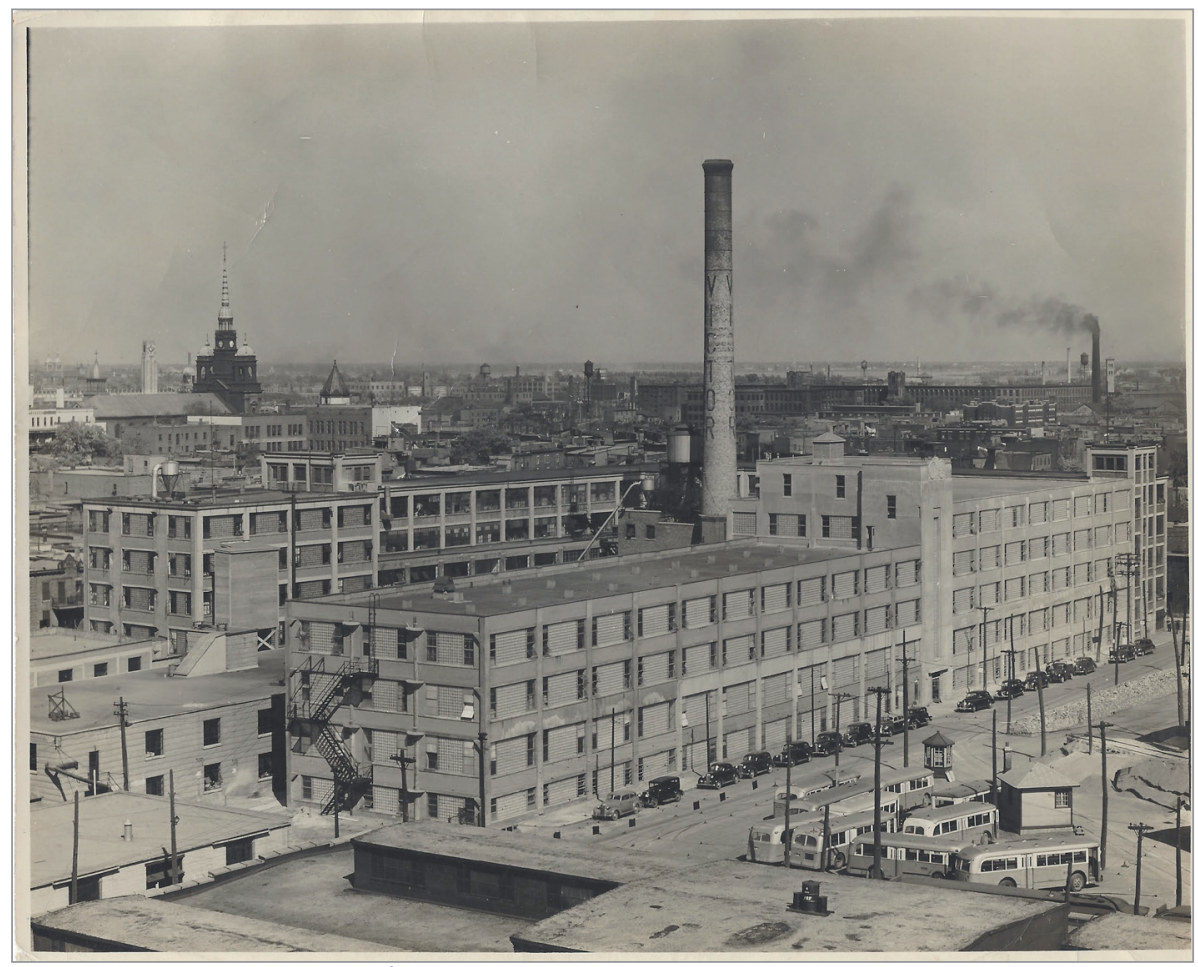

ILL. 1. VUE DE L'USINE RCA-VICTOR APRÈS L'AGRANDISSEMENT DE 1943. | MUSÉE DES ONDES BERLINER, S.D.

\section{$>$ LAURENT DAMBRE- SAUVAGE}

'édifice RCA (ill. 1), situé dans I'arrondissement montréalais du Sud-Ouest, à la limite nord du quartier Saint-Henri, accueille actuellement diverses entreprises, des ateliers d'artistes et le Musée des ondes Emile Berliner (MOEB) ${ }^{1}$. Représentatif de l'architecture moderniste à Montréal, il est le témoin d'un riche passé industriel. Il a, en effet, été le lieu de la fabrication des premiers gramophones en Amérique, a par la suite abrité les activités de la célèbre compagnie RCAVictor avant de connaître une phase de déclin. Immeuble d'intérêt patrimonial, il est l'objet d'un redéveloppement immobilier porté par des acteurs privés en vue $d^{\prime} y$ installer diverses entreprises. À ce projet patrimonial s'articule celui porté par le MOEB qui vise à mettre en valeur I'histoire du bâtiment et de l'industrie qu'il a abritée. Au regard du développement socioéconomique du territoire dans lequel il est inséré, la lecture de ce cas soulève plusieurs questions : Comment un projet construit sur des logiques privées contribue-t-il au développement du quartier? Un projet piloté par un petit nombre d'acteurs peut-il instituer la mise en place de dynamiques communautaires et, plus particulièrement, favoriser l'émergence d'une communauté patrimoniale? La présence dans un même bâtiment de plusieurs projets patrimoniaux induit-elle des dynamiques de concurrence et, dans ce cas, comment les différents projets s'articulent-ils les uns aux autres?

Pour répondre à ces questions, nous avons mené une recherche qui s'est déroulée en trois temps. En premier lieu, nous avons procédé à une revue de presse à partir d'articles repérés dans la base de 
données Eureka. Celle-ci nous a permis de sélectionner soixante-quinze articles véhiculant diverses représentations qui ont apporté des réponses à nos questions. En deuxième lieu, nous avons effectué, le 17 février 2018, une première visite du bâtiment et du MOEB, ce qui nous a permis de relever les noms des entreprises et des individus qui y sont installés. Nous avons alors constitué une base de données à partir d'informations recueillies via le moteur de recherche Google. Cette base rassemble des renseignements sur les locataires de l'immeuble (domaine d'activité, site Internet, page Facebook, coordonnées). Enfin, nous avons mené dix entretiens semi-dirigés avec l'un des propriétaires du bâtiment, la directrice du MOEB, deux habitants du quartier, des membres de la Société historique de Saint-Henri, des chefs et des employés d'entreprise (4 entrevues) et des artistes (2 entrevues).

Dans une première partie, nous présenterons l'écosystème dans lequel s'insère I'édifice RCA. Ensuite, nous analyserons l'économie de ce projet en exposant la stratégie mise en place par les acteurs et en analysant les valeurs de développement (valeur communautaire et valeur de reconnaissance) mobilisées, ou non, ici. Enfin, en partant des forces et des faiblesses identifiées dans ce projet, nous proposerons un plan d'action permettant à la fois de maximiser l'apport du projet au développement socioéconomique du quartier et d'assurer la préservation, dans le temps, de l'objet patrimonial en question.

\section{ÉCOLOGIE DE L'ÉDIFICE RCA}

Lucie K. Morisset ${ }^{2}$ définit l'écologie d'un projet patrimonial comme l'ensemble des «acteurs, en un milieu donné » produisant un discours d'authenticité qui qualifie un objet particulier, lui conférant ainsi une

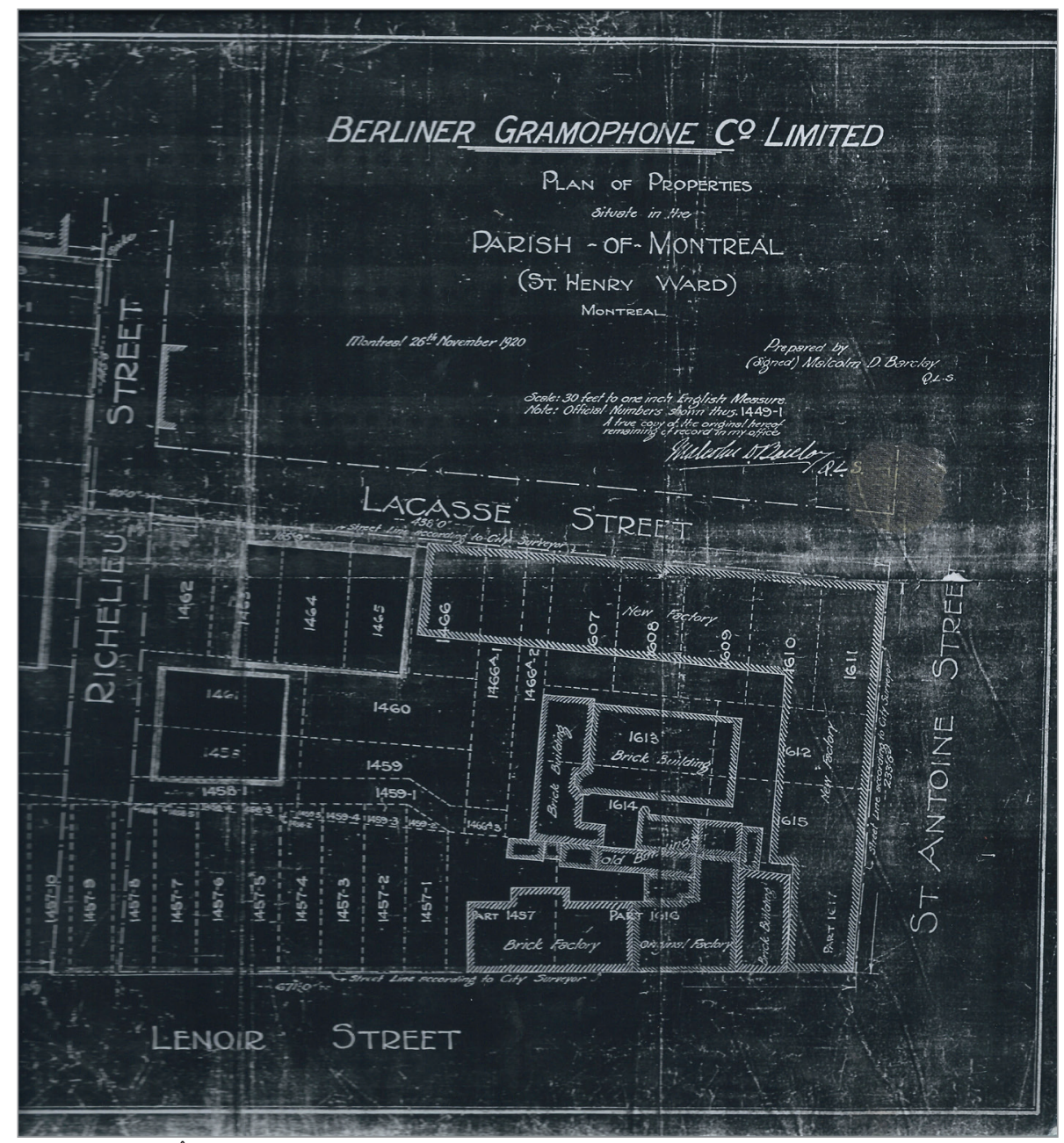

ILL. 2. PLAN DES BÂTIMENTS DE L'USINE BERLINER GRAMOPHONE EN 1920. | MUSÉE DES ONDES BERLINER, S.D.

valeur spécifique qui en change l'état. Nous voulons, dans cette partie, revenir sur les différentes composantes de l'écosystème entourant l'édifice RCA et décrire I'objet en lui-même, dans ses dimensions physiques et historiques, les représentations qui lui sont attachées, les principaux acteurs associés au projet patrimonial et les moyens sur lesquels il prend appui.

\section{L'édifice RCA}

Ancien complexe industriel consacré à l'industrie de la musique (enregistrement, pressage de disques, fabrication d'appareil d'écoute) puis à la fabrication d'appareils de télécommunication de haute technologie, l'édifice RCA est constitué d'un ensemble de bâtiments en « $U$ » dont la construction s'échelonne de 1920 à 1943 (ill. 2), les bâtiments originaux datant de 1908 ayant été démolis lors de l'agrandissement du complexe dans les années 1940. Représentant un des premiers exemples d'architecture industrielle moderniste à Montréal, l'ensemble est remarquable par l'unité architecturale des façades sur rue (ill. 3). Construit en 1920 sur les plans des architectes Donald Norman, MacVicar et John Charles 


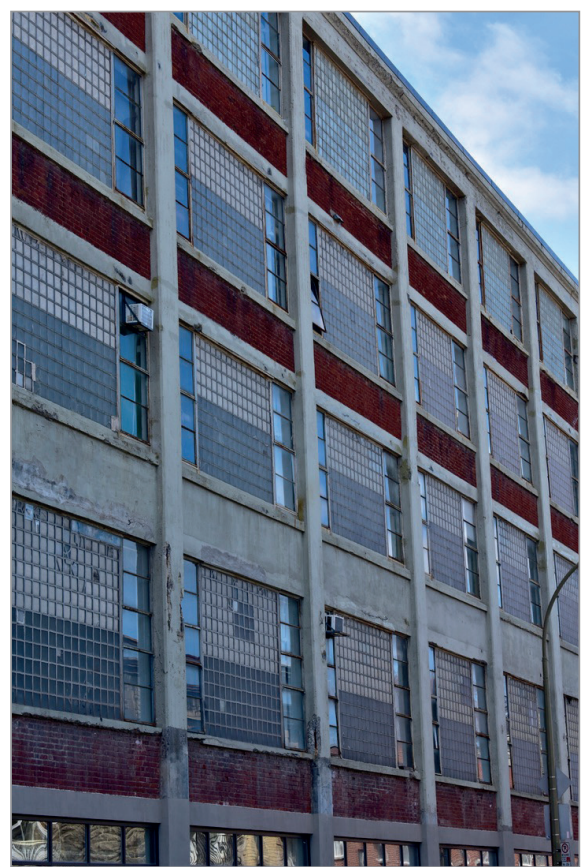

ILL. 3. BÂTIMENT RCA - FAÇADE SUR LA RUE LACASSE. | LAURENT DAMBRE-SAUVAGE, 9 AVRIL 2018

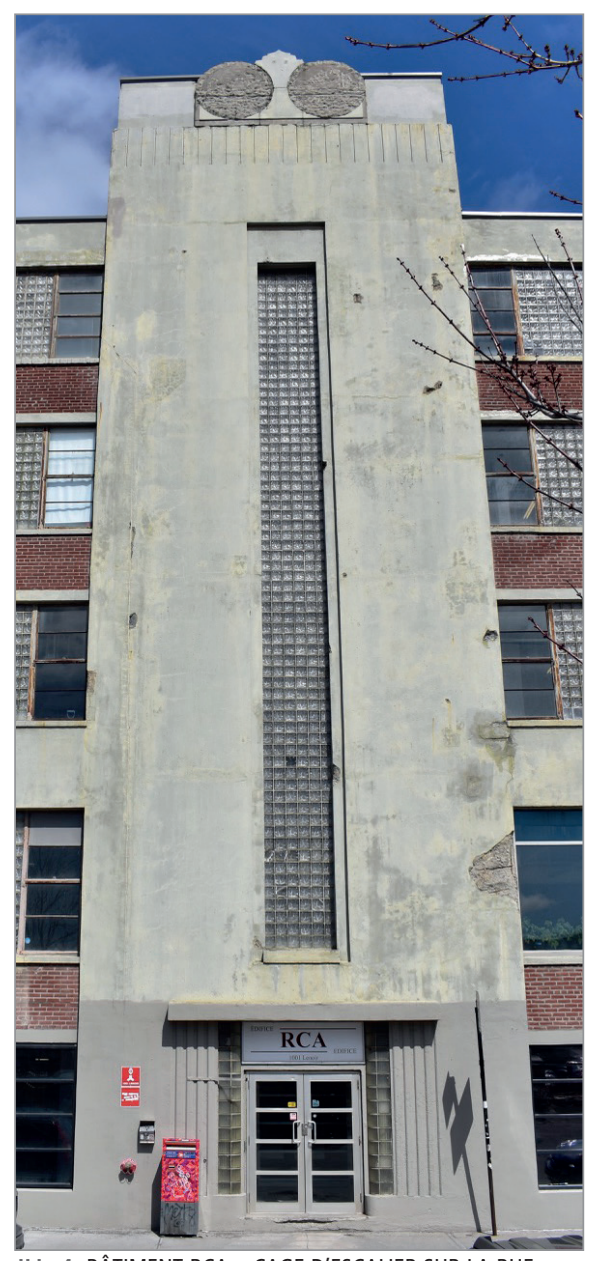

ILL. 4. BÂTIMENT RCA - CAGE D'ESCALIER SUR LA RUE LENOIR. | LAURENT DAMBRE-SAUVAGE, 9 AVRIL 2018.

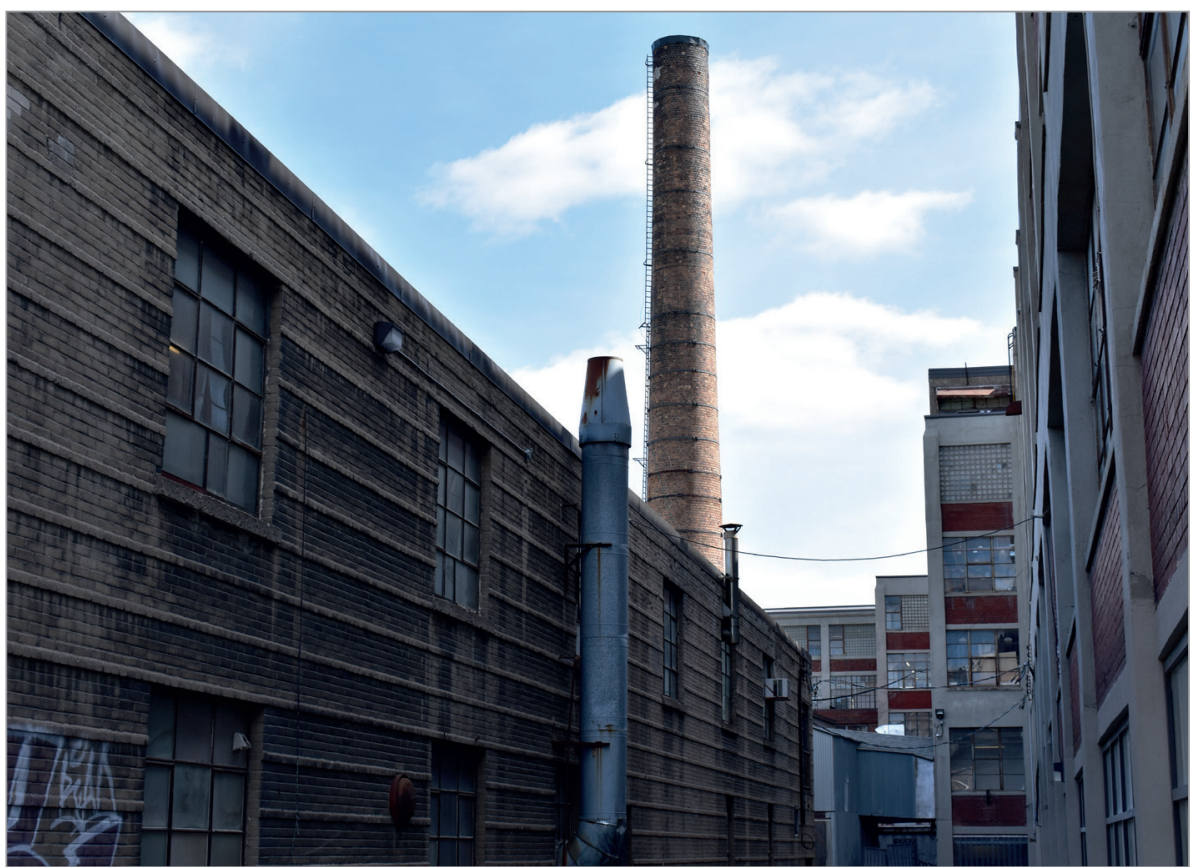

ILL. 5. BÂTIMENT RCA - VUE DE LA COUR SUR LA CHAUFFERIE ET LA CHEMINÉE. | LAURENT DAMBRE-SAUVAGE, 9 AVRIL 2018.

Allison Hériot, un premier bâtiment en « $L$ » à l'angle sud-est de l'ensemble impose un schéma qui sera repris pour les autres façades sur rue : la structure en béton demeure extrêmement lisible et quadrille fortement l'élévation, tout en permettant une large fenestration; elle scande avec force les verticales, les lignes horizontales étant, quant à elles, soulignées par un parement de brique introduisant une polychromie qui adoucit l'ensemble. La façade sur la rue Lenoir, à I'ouest du bâtiment, construite en 1942 par W.K. Gordon Lyman, introduit à ce schéma une cage d'escalier qui non seulement souligne l'entrée du bâtiment, mais également rompt la répétition du rythme puissant imposé par la structure (ill. 4). Elle présente des éléments décoratifs art déco et arborait dans des médaillons les emblèmes, aujourd'hui disparus, de la compagnie. L'ensemble est complété par d'autres bâtiments au cœur desquels la chaufferie comporte une haute cheminée (53 mètres) qui domine le tout (ill. 5).

Sur le plan technique, il présente également des éléments intéressants : le système de renforcement du béton, dit «mushroom cap column system », permet une large fenestration et une modulation aisée des espaces intérieurs. Le bâtiment abrite un studio d'enregistrement, conçu par la firme RCA-Victor en 1943, dans lequel le revêtement des murs composé de panneaux de bois polycylindriques assure des performances acoustiques exceptionnelles. II s'agit d'un des derniers studios d'enregistrement datant de la première moitié du vingtième siècle encore en fonction (ill. 6).

Au-delà de ces caractéristiques physiques, I'histoire même du lieu concourt à sa valeur. En effet, le bâtiment est associé à la figure d'Emile Berliner [1851-1929], inventeur du gramophone (1888) (ill. 7). Il installa sa compagnie, la Gramophone Berliner Company, à Montréal en 1900 et fera construire les premiers bâtiments du complexe dès 1908 (ill. 8). Si son invention en fait un personnage important dans I'histoire mondiale de la musique, il est également, avec son fils, à l'origine des premiers enregistrements d'artistes québécois ${ }^{3}$. 


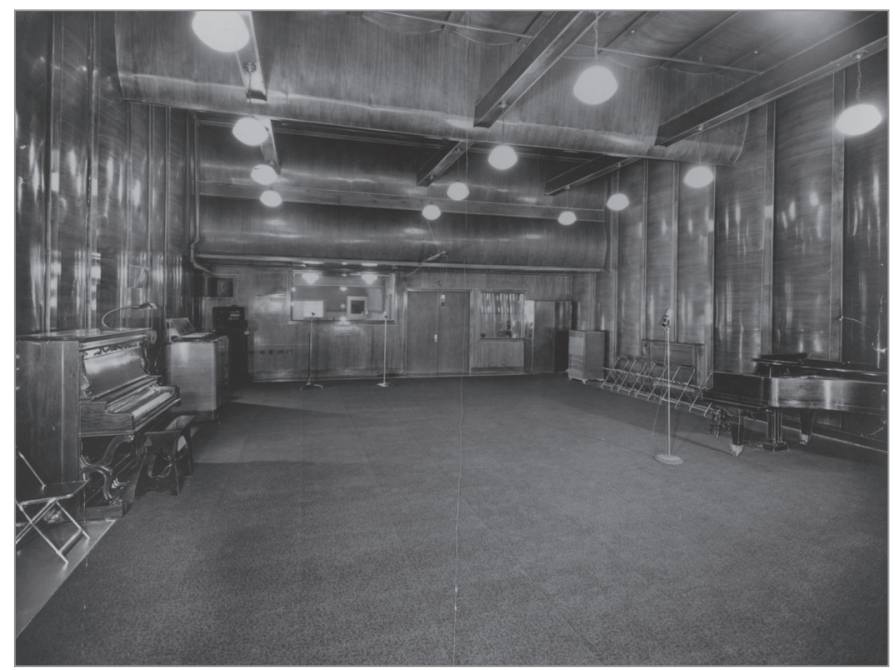

ILL. 6. LE STUDIO D'ENREGISTREMENT RCA-VICTOR AU DÉBUT DES ANNÉES 1940. | CANADIAN SCIENCE AND TECHNOLOGY MUSEUM (INGENIUM), S.D.

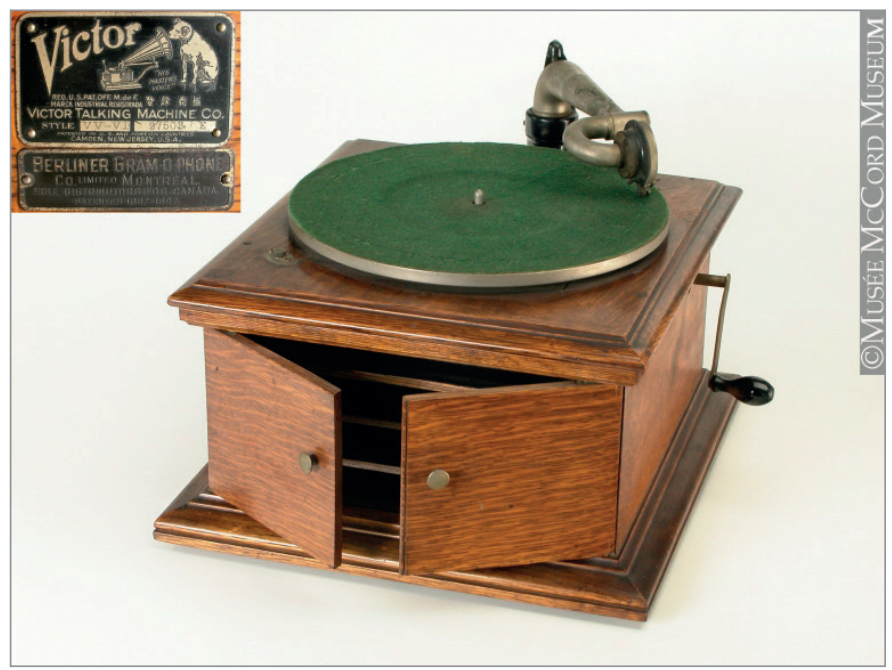

ILL. 7. TOURNE-DISQUE, VICTOR TALKING MACHINE CO, 1902-1925. | ๑ MUSÉE MCCORD.

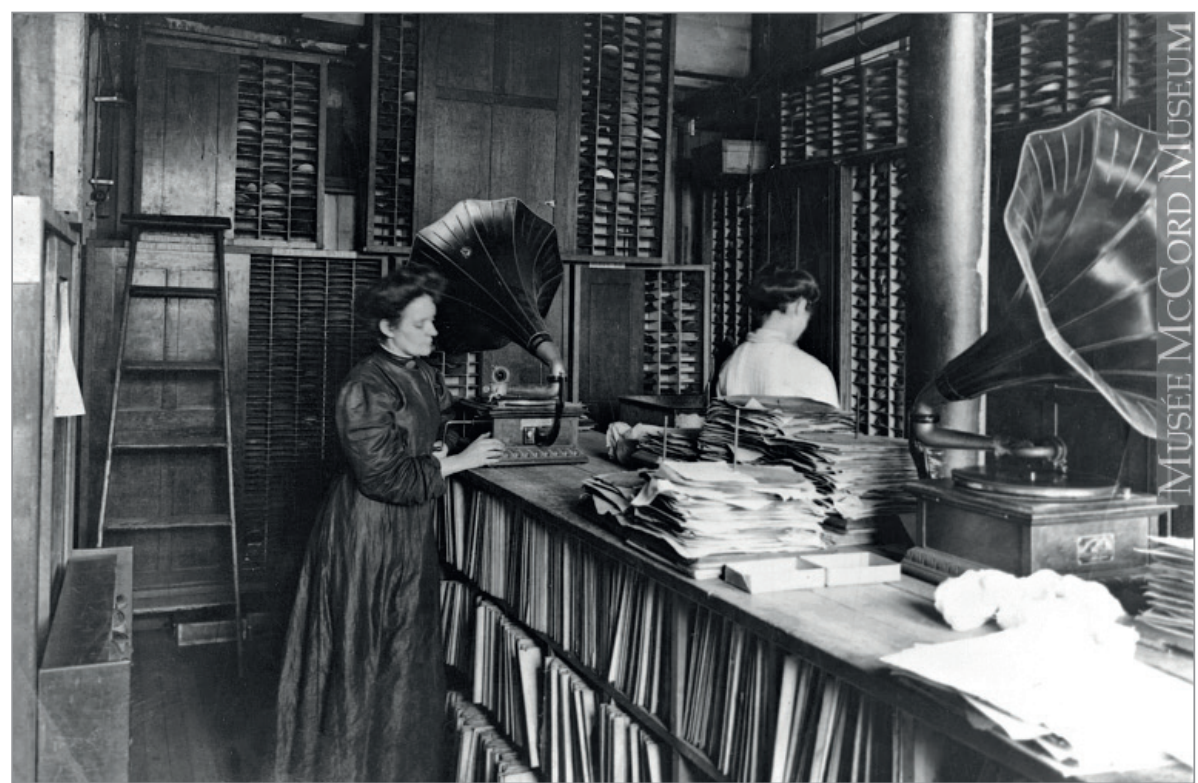

ILL. 8. SALLE DES MATRICES, BERLINER GRAMOPHONE COMPANY, MONTRÉAL, QC, 1910. | ๑ MUSÉE MCCORD.

Après sa fondation, I'histoire de I'usine se structure en trois phases. La première correspond à la période allant jusque 1929 où l'usine est rachetée par la compagnie RCA-Victor, qui voit la construction des premiers bâtiments. Durant la seconde phase, de 1923 à 1978, RCA agrandit l'ensemble et construit le studio d'enregistrement. Pendant la guerre froide, on y développe des technologies de surveillance et y construit le premier satellite canadien. En 1966, le pressage de disques est abandonné. RCA quittera les lieux en 1978, ouvrant alors la troisième phase marquée par une discontinuité non seulement quant à l'usage du bâtiment mais également quant à sa valeur symbolique liée à l'aventure industrielle qu'il a portée.
L'immeuble est alors occupé par diverses entreprises et abrite des ateliers d'artistes; son état de conservation se détériore peu à peu. En 1985, le Studio Victor restaure l'ancien studio et y relance les enregistrements, plus particulièrement d'artistes québécois. Le MOEB s'y installe en 1996. En 2004, le propriétaire de l'époque tente de transformer le lieu en immeuble en copropriété et en commerces, sans succès; il vendra le complexe aux propriétaires actuels en 2008. Enfin, l'année 2015 voit la fermeture du Studio Victor qui laisse place, en 2017, à la Hacienda Creative, entreprise qui élabore des projets musicaux, principalement pour l'industrie du jeu vidéo.

Il convient, au terme de ce survol de l'histoire du bâtiment, de souligner l'absence de crise patrimoniale. La discontinuité survenue au tournant des années 1980 n'a en effet pas conduit à une mise en danger du bâtiment.

\section{Les représentations attachées à l'édifice RCA}

Au fil du temps, I'histoire du bâtiment s'est cristallisée dans un certain nombre de représentations relevées dans la 
presse. La première met en avant le bâtiment comme fleuron de l'industrie canadienne. La figure d'Emile Berliner est présentée comme celle d'un visionnaire ${ }^{4}$ et d'un pionnier ${ }^{5}$ initiant une aventure industrielle qui contribue à faire du bâtiment et du quartier Saint-Henri un "centre névralgique de l'industrie du disque en Amérique du Nord ${ }^{6}$ ", voire une «Silicon Valley du début du $20^{e}$ siècle ${ }^{7}$ ". En effet, le développement d'appareils de télécommunication et de satellites fait du bâtiment " un des pôles centraux des télécommunications au Canada " et met le quartier «à la fine pointe du développement de la communication de masse ${ }^{8}$.

Plus spécifiquement, c'est principalement le rôle du bâtiment dans l'industrie musicale qui est mis en avant. D'une part, il s'agit du lieu où ont été créés les premiers gramophones en Amérique (ill. 8); d'autre part, le studio d'enregistrement constitue un jalon de l'histoire de la musique, autant par ses qualités techniques (il est " une sorte de stradivarius de l'enregistrement $^{9}$ ») que par le prestige des artistes qui y ont joué ${ }^{10}$. Il est ainsi un lieu «emblématique de la communauté musicale ${ }^{11} »$.

L'édifice est également vu comme un lieu de référence pour différentes communautés. Le MOEB constitue un point de ralliement pour les anciens employés de I'usine RCA qui y organisent de «joyeuses retrouvailles ${ }^{12}$ » ou pour des passionnés de technologie qui se réunissent «pour réparer télévisions, amplificateurs et radios anciennes ${ }^{13}$ ". Il permet également d'éclairer les habitants sur I'histoire de leur quartier ${ }^{14}$. Enfin, le bâtiment est présenté comme abritant une communauté particulièrement dynamique d'artistes et d'artisans ${ }^{15}$.

Jamais menacé de destruction, le RCA n'a pas proprement fait l'objet d'une quelconque crise patrimoniale. Cependant, la presse s'est fait l'écho de deux moments de tension dans I'histoire du bâtiment. Le premier survient au tournant des années 1990-2000 où se pose la question de I'usage de lieux dont l'état se dégrade petit à petit ${ }^{16}$. Le propriétaire d'alors a envisagé de transformer le bâtiment en immeuble de commerces et d'appartements, ce qui nécessitait un changement de zonage, refusé par la Mairie pour des raisons plus ou moins frauduleuses ${ }^{17}$. Le second est lié à la fermeture du Studio Victor en 2015. À cette occasion, la presse multiplie les articles mettant en avant son caractère «mythique $»^{18}$. Contrairement à la première phase de tension évoquée précédemment, on voit apparaître à cette occasion des références au caractère patrimonial de l'édifice, marquant la volonté des acteurs de véhiculer une valeur particulière qui y serait attachée dans le but d'en préserver à la fois l'usage et l'intégrité. Ainsi, son directeur affirme : «Ce studio, c'est un patrimoine important pour Saint-Henri, le Québec et le Canada ${ }^{19}$." Le dénouement de cette crise permet de mettre en avant le rôle des propriétaires dans la préservation de ce patrimoine ${ }^{20}$ et une certaine continuité d'usage permise par l'installation dans les locaux d'une compagnie œuvrant dans la musique ${ }^{21}$.

\section{Les acteurs de l'écosystème RCA}

Un certain nombre d'acteurs dont nous présentons ici les principaux gravitent autour du bâtiment RCA. Le MOEB est historiquement le premier d'entre eux. Le musée a été créé en 1992 par Gaétan Pilon, qui dirigeait le Studio Victor, et des membres de la Société historique de SaintHenri, avec la mission de «sauvegarder, étudier et diffuser le patrimoine matériel et immatériel associé à I'histoire culturelle, architecturale, technologique et scientifique de l'industrie des ondes sonores. L'institution porte un intérêt particulier à Emile Berliner, aux entreprises nées de son travail et à leur impact local, national et international ${ }^{22}$ ». Le MOEB s'installe dans le bâtiment où il occupera plusieurs locaux au fil du temps. Dès lors, des dons venant d'habitants du quartier ou de collectionneurs vont lui permettre de constituer une collection riche d'environ trente mille objets construite autour de la thématique des techniques du son et de l'image. Le musée est structuré en organisme sans but lucratif (OSBL).

Il promeut le bâtiment et l'histoire industrielle dont il est le témoin comme un élément important du patrimoine canadien. Le musée a développé, en 2015, un projet de "Cité des ondes» avec l'ambition d'augmenter la superficie du musée, de le mettre aux normes de conservation, d'engager une grande campagne de restauration des collections et de racheter une partie du bâtiment contenant le studio d'enregistrement afin de le restaurer et de l'intégrer au musée ${ }^{23}$. Ce projet ne pourra malheureusement pas se concrétiser, faute de financement.

Arrivés dans l'écosystème du RCA en 2009 , les quatre propriétaires actuels ${ }^{24} y$ jouent un rôle fondamental. Ils ont acheté le bâtiment pour la somme de dix-huit millions et demi de dollars dans le but de le redévelopper. Parmi eux, Georges Coulombe est plus particulièrement mis en avant dans la presse ${ }^{25}$. À la tête d'un groupe de gestion immobilière, celui-ci est passionné par le patrimoine architectural québécois qu'il s'emploie à restaurer, à préserver et à moderniser. Son engagement pour le patrimoine lui a valu, en 2008, le Prix d'excellence de la Société immobilière Trans-Québec (SITQ), « attribué à une personne ou à un groupe qui a démontré son désir de contribuer à la préservation du patrimoine dans l'intérêt commun ${ }^{26}$ ". Cette passion du patrimoine est partagée par les autres membres de l'équipe de propriétaires, qui portent 
également une attention particulière au développement durable : la préservation d'un bâtiment plutôt que sa destruction s'inscrit pour eux dans une démarche écologique affirmée.

L'achat du bâtiment par ces investisseurs à concouru à rassurer les acteurs locaux et plus particulièrement les membres de la Société historique de Saint-Henri sur l'avenir de l'édifice RCA ${ }^{27}$.

Il faut également compter les entreprises, organismes et individus locataires d'espaces dans le bâtiment parmi les acteurs de l'écosystème du RCA. Le panneau situé dans l'accueil de l'édifice permet d'en dénombrer cent trente-huit ${ }^{28}$. Ceux-ci sont extrêmement divers, que ce soit par leur taille, leur secteur d'activité ou leur stade de développement. Les secteurs de I'économie culturelle (artistes, galeries, festivals...) et créative (jeux vidéo, photographes, designers, intelligence artificielle...) sont numériquement les mieux représentés (81 organismes). Parmi eux, la Hacienda Creative occupe le studio d'enregistrement. Elle a été fondée en 2007 et regroupe dix "artisans sonores" qui élaborent des projets musicaux pour les jeux vidéo, la publicité ou encore les musées. Son installation dans le studio est l'occasion pour les dirigeants de l'entreprise de témoigner d'une attention particulière portée au caractère patrimonial d'un immeuble présenté comme "un des bâtiments les plus importants de l'industrie du son et de la musique ${ }^{29}$ », inscrivant ainsi l'activité de l'entreprise dans une histoire mythifiée, tout en tirant profit des qualités techniques du studio. Cette installation a donné l'occasion d'une restauration, financée à parts égales par les propriétaires et les nouveaux locataires (à hauteur de $250000 \$$ chacun), dans le but de «saisir l'essence historique de l'espace $^{30}$ ». Cette installation constitue par ailleurs un projet concurrent à celui porté par le MOEB auquel elle semble mettre un terme plus ou moins définitif.

La concurrence de projets nous invite à nous questionner sur les ressources dont disposent les différents acteurs. Elles constituent un facteur important dans la réalisation des projets patrimoniaux, leur permettant de se concrétiser ou provoquant leur suspension.

\section{Les moyens}

Le projet du RCA prend appui sur deux types de ressources. En premier lieu, les moyens financiers mobilisés sont principalement des moyens privés constitués par l'investissement des propriétaires relayé, dans le cas du studio d'enregistrement, par celui d'entreprises locataires.

De son côté, le MOEB bénéficie de subventions qui sont octroyées sur projet (principalement pour ses expositions). En outre, depuis 2016, I'Arrondissement du Sud-Ouest s'est engagé à appuyer le fonctionnement du musée à hauteur de dix mille dollars par an pendant trois ans.

En second lieu, les moyens administratifs appuyant le projet sont assez faibles. L'immeuble n'est pas inscrit au Registre du patrimoine culturel, ni au Répertoire canadien des lieux patrimoniaux. Son importance patrimoniale est cependant prise en compte dans divers outils de diffusion de la connaissance. Ainsi, il est inventorié dans le Répertoire du patrimoine culturel du Québec. Il apparaît également comme un «immeuble patrimonial de valeur exceptionnelle" dans une évaluation du patrimoine urbain de I'arrondissement du Sud-Ouest ${ }^{31}$. Enfin, il fait l'objet d'un énoncé d'intérêt patrimonial (16 avril 2012). Finalement, c'est son identification comme bâtiment d'intérêt patrimonial et architectural dans le Plan d'urbanisme qui lui assure une certaine protection aux termes des règles d'urbanisme de la Ville de Montréal.

\section{ÉCONOMIE DE L’ÉDIFICE RCA}

Exposer l'économie d'un projet patrimonial, c'est révéler les moyens qui sont mis en œuvre pour en assurer la concrétisation. Nous allons, dans cette partie, analyser la stratégie développée par les acteurs, analyser les effets de cette stratégie sur le développement du territoire et discuter de la mobilisation des valeurs de développement permise par cette stratégie.

\section{Une stratégie fondée}

sur un dynamique capitaliste

Ce projet est directement porté par le consortium de quatre propriétaires. La stratégie qu'ils ont mise en place est construite sur une dynamique capitaliste dans laquelle les profits générés par la location d'espaces aux entreprises - profits qui sont donc tirés de I'usage du bâtiment - sont, en partie, réinvestis dans sa préservation et sa restauration. Ce réinvestissement permet une double augmentation. D'une part les améliorations apportées au bâtiment rendent les espaces loués plus désirables et mieux adaptés aux besoins des locataires, accroissant la valeur d'usage et conséquemment la valeur locative. D'autre part, le capital réinvesti dans le bâtiment en accroît la valeur d'échange, c'est-à-dire le prix sur le marché.

À l'issue de cette dynamique, les besoins des locataires sont réévalués. Si le besoin premier est l'accès à des espaces bon marché - soulignons ici que le mauvais état initial du bâtiment et son emplacement dans un quartier plutôt dévitalisé concouraient au prix modique des espaces -, le bâtiment apparaîtra alors moins adapté et le locataire pourra être amené à quitter 
les lieux pour laisser place à des locataires mieux nantis qui, à leur tour, contribueront à leur revalorisation.

L'avantage de ce modèle circulaire et cumulatif réside dans son caractère autosuffisant : les revenus produits par la marchandisation de l'usage du lieu suffisent à sa préservation. II comporte néanmoins un certain nombre d'inconvénients. D'abord, cette stratégie nécessite un investissement initial conséquent. Dans le cas d'un bâtiment patrimonial, cet investissement peut néanmoins se révéler avantageux pour un investisseur disposant d'importants moyens financiers: "Je suis persuadé que j'ai choisi la meilleure niche à long terme [dit Coulombe]. À Montréal, c'est encore possible d'acheter, de rénover et de louer à bon prix. Construire des immeubles intéressants, ça coûte 250 ou 300 \$ le pied carré. Moi, rénover me coûte moitié moins ${ }^{32}$."

Ensuite, dans ce modèle, le caractère patrimonial de l'immeuble apparaît comme secondaire, c'est-à-dire que la valeur d'usage prime sur les valeurs d'existence instituant le caractère patrimonial d'un objet architectural (valeurs d'âge, d'art, de position et de matérialité). Le sous-investissement de ces valeurs d'existence pourrait, à terme, nuire à la préservation d'un bâtiment rendu quelconque. Ici, seule la passion des propriétaires garantit la préservation du bâtiment.

Finalement, il convient de relever l'instabilité de ce modèle à plusieurs égards. Instabilité tout d'abord au regard de la propriété : en effet, il est possible que les propriétaires actuels puissent vendre l'immeuble à des personnes n'ayant pas de sensibilité particulière envers son caractère patrimonial, ce qui, à plus ou moins long terme, peut constituer une mise en danger du bâtiment. Instabilité ensuite

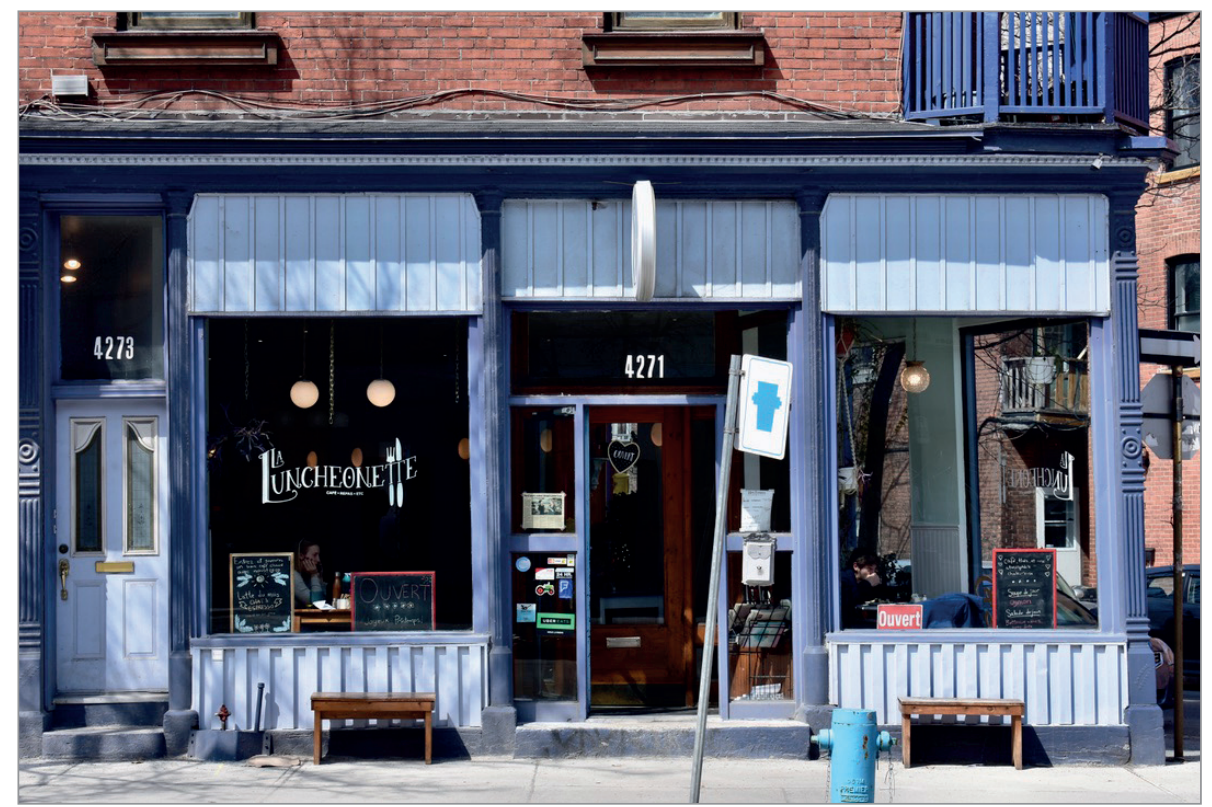

ILL. 9. COMMERCE RUE SAINT-JACQUES. | LAURENT DAMBRE-SAUVAGE, 9 AVRIL 2018.

au regard des locataires dans la mesure où le modèle peut conduire à l'éviction de ceux qui sont les plus fragiles économiquement et à une rotation importante des entreprises si elles s'inscrivent dans une logique purement comptable, quittant le bâtiment si une opportunité plus avantageuse se présente ailleurs.

\section{Apport au développement local}

Malgré ces inconvénients, le modèle développé par l'écosystème du RCA parvient à engager le projet dans une dialectique porteuse de développement socioéconomique avec son territoire. Tout d'abord le projet a permis l'installation d'un nombre important d'entreprises et, ainsi, la création d'emplois : environ mille cinq cents personnes travaillent effectivement dans le bâtiment. En outre, I'arrivée d'entreprises œuvrant dans des secteurs demandant une main-d'œuvre hautement qualifiée entraîne l'installation dans le quartier de professionnels appartenant à des catégories socioprofessionnelles supérieures, ce qui concourt à la valorisation foncière de l'environnement. De plus, cet afflux de professionnels a des effets positifs sur le tissu commercial du quartier (notamment l'ouverture de nouveaux commerces) (ill. 9).

Enfin, l'installation d'entreprises et l'augmentation de la valeur d'échange du bâtiment se traduisent par l'accroissement des taxes et donc l'augmentation de revenus pour la Municipalité, revenus qui peuvent être mis au profit du développement de nouveaux services dans le quartier, autrement dit, qui accroissent la valeur publique du bâtiment.

Par ailleurs, il faut souligner que la stratégie développée ici est maximisée par le développement du quartier. En effet, le RCA est situé à la marge d'un quartier - Saint-Henri - dont la partie sud a été revitalisée ${ }^{33}$. Le projet offre ainsi un avantage compétitif important par rapport aux concurrents situés plus au sud, tout en étant inséré dans un territoire qui comporte un certain nombre de commodités (proximité du métro, du 

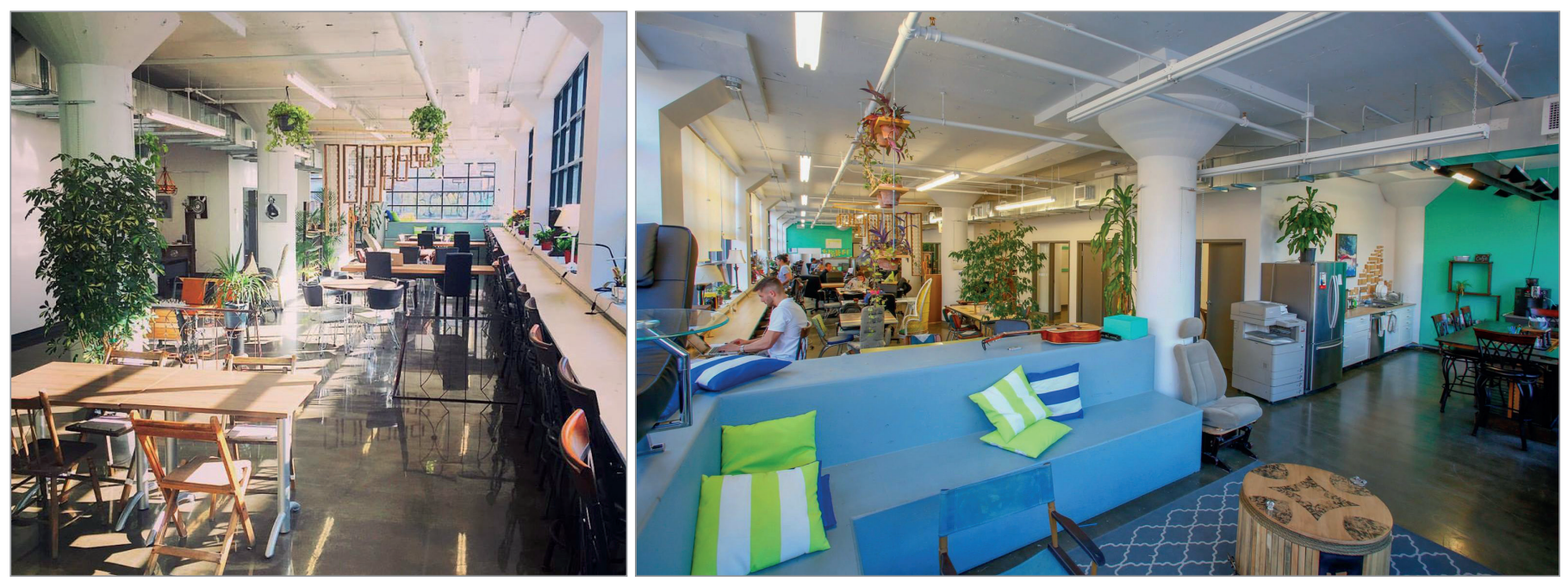

ILL. 10 A ET B. ESPACE DE COWORKING, LE TABLEAU BLANC. | TABLEAU BLANC, S.D.

centre-ville, de zones d'activité commerçante ou récréotouristique).

Dans le cas du RCA, la valeur d'usage du bâtiment prime donc sur des valeurs d'existence qui sont, toutefois, affirmées et défendues par les propriétaires et le MOEB : si l'usage permet le maintien du bâtiment dans un état fonctionnel, I'engagement patrimonial des propriétaires en garantit la préservation. Toutefois, dans un contexte post-moderne où les références communes sont atomisées, la préservation du patrimoine à long terme repose sur l'érection de ce dernier en commun ${ }^{34}$, c'est-à-dire en objet approprié symboliquement ou littéralement par une communauté pour laquelle il devient une ressource. Le patrimoine devient alors porteur de valeurs dynamiques qui en assurent la préservation dans la durée tout en agissant de manière positive sur la communauté qui se saisit d'un bâtiment pour l'investir d'une valeur particulière, qui ne lui est en aucun cas ontologique, enclenchant un processus de patrimonialisation. Ces valeurs dynamiques sont la valeur communautaire, mesurant la cohésion de la communauté créée par le processus de patrimonialisation, et la valeur de reconnaissance, mesurant la tangibilité de la mémoire patrimoniale.

Les valeurs dynamiques produites par le RCA

\section{La valeur communautaire}

La concentration d'entreprises d'économie culturelle et créative dans un bâtiment patrimonial nous a incité à rechercher ici la présence de deux communautés : une communauté entrepreneuriale et une communauté patrimoniale.

Une communauté entrepreneuriale? Dans le cadre de l'économie du savoir, l'information constitue, pour des entreprises localisées dans une même aire géographique, un commun intangible ou, pour le dire autrement, un stock de ressources dans lequel ces entreprises peuvent puiser pour nourrir leurs pratiques professionnelles. Plus particulièrement, les échanges formels et informels permis par la proximité peuvent nourrir des processus d'innovation à la base de la création de valeur pour les entreprises culturelles/créatives, constituant de ce fait des "districts créatifs ${ }^{35}$ ". Se pose alors la question de savoir si les relations entre les entreprises localisées dans l'édifice RCA génèrent de la plus-value, directe pour les entreprises et indirecte pour les propriétaires de l'immeuble.

Les entretiens que nous avons menés révèlent certes la présence d'échanges et de collaboration entre les entreprises du RCA, mais ceux-ci sont extrêmement limités, voire "anecdotiques" pour reprendre l'expression d'un répondant. Dans leur grande majorité, les personnes qui travaillent dans le RCA sortent peu de leur entreprise dans le courant de la journée et n'échangent pas avec les autres acteurs situés dans le bâtiment.

Pourtant, un certain nombre de facteurs pourraient contribuer à faciliter les échanges entre les divers acteurs. Premièrement, un espace de coworking, Le Tableau Blanc, installé au rez-de-chaussée du bâtiment, est ouvert à accueillir des événements destinés aux entreprises de l'immeuble (ill. 10a et b). Il organise, par exemple, des expositions et des « 5 à 7 » de réseautage. Par ailleurs, la formule du coworking incite intrinsèquement à développer des collaborations ${ }^{36}$. 


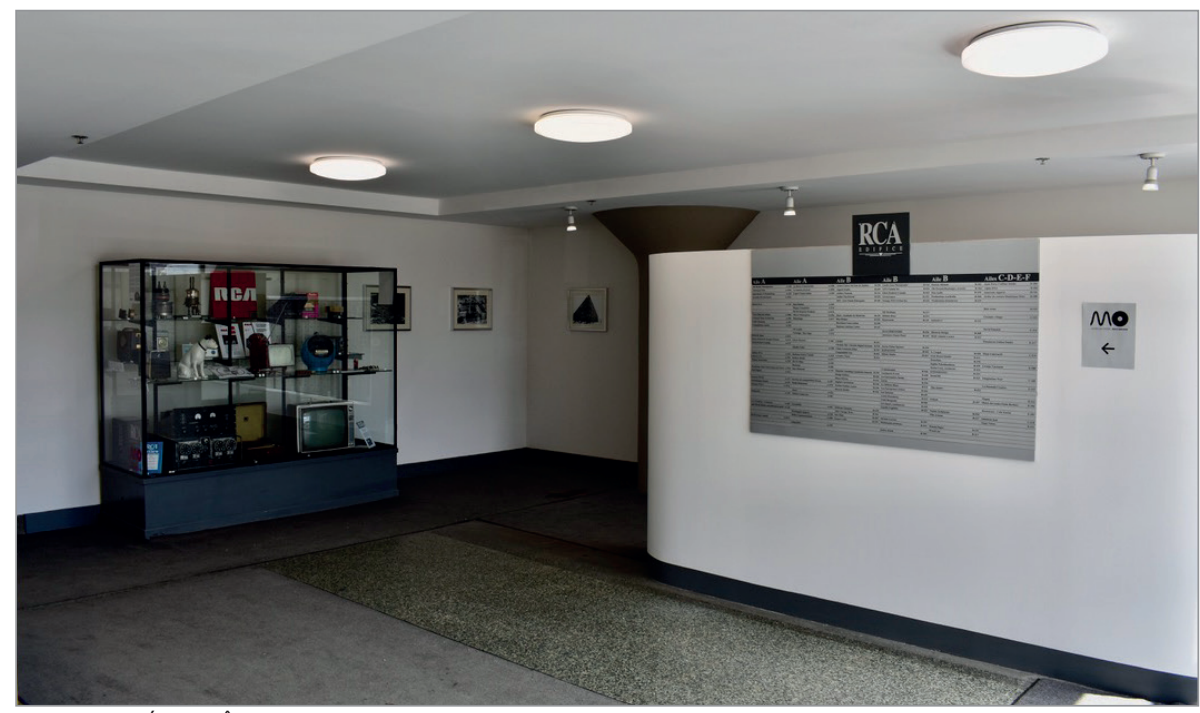

ILL. 11. ENTRÉE DU BÂTIMENT. | LAURENT DAMBRE-SAUVAGE, 9 AVRIL 2018.

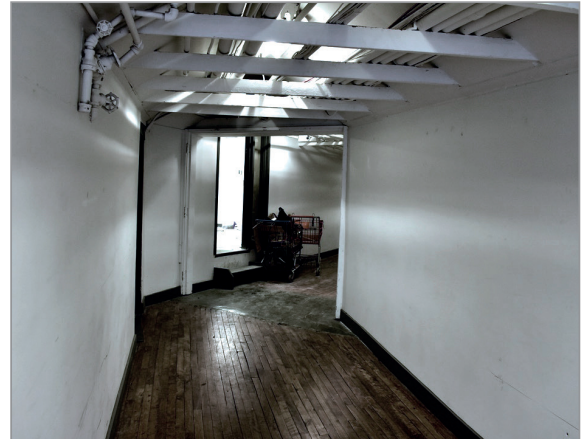

ILL. 12. COULOIRS À L'INTÉRIEUR DU BÂTIMENT. | LAURENT DAMBRE-SAUVAGE, 9 AVRIL 2018.

Deuxièmement, certains acteurs manifestent une volonté d'organiser des événements rassembleurs. Les propriétaires, par exemple, ont déjà organisé des apéritifs et proposent de participer à l'organisation des événements qui se tiennent dans le bâtiment. Lors de son installation, La Hacienda Creative a, quant à elle, organisé un barbecue sur le toit du bâtiment et une visite du studio ouverte aux entreprises du bâtiment.

Il existe, au sein du bâtiment, des espaces qui pourraient favoriser les rencontres et les interactions (Café Zinzin, galeries, musée, gym...). Or, plusieurs facteurs

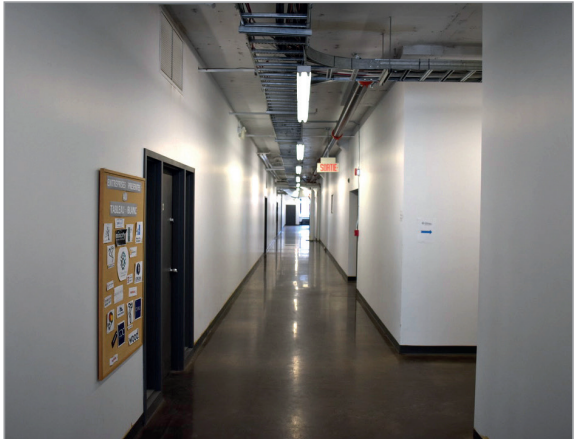

ILL. 13. COULOIRS À L'INTÉRIEUR DU BÂTIMENT. | LAURENT DAMBRE-SAUVAGE, 9 AVRIL 2018.

peuvent expliquer l'absence d'interactions. Les travailleurs et les chefs d'entreprise rencontrés font état de charges de travail importantes qui laissent peu de place à la socialisation. Lorsque du temps est libéré, il est en priorité mis à profit du développement de relations internes à l'entreprise (team building).

Il faut également souligner le rôle inhibant de la configuration d'espaces internes peu propices aux échanges, avec l'omniprésence des couloirs et les nombreuses entrées indépendantes (ill. 11, 12 et 13). Par ailleurs, les espaces qui pourraient être conviviaux (restaurants, musée, galeries) se trouvent au cœur du bâtiment, au détour de couloirs dédalesques qui les rendent invisibles. Il est à noter que la dynamique économique mise en œuvre dans ce projet freine le développement d'espaces de convivialité ouverts à tous en incitant à maximiser le nombre d'espaces rentables: on a alors tendance à réduire au maximum les espaces de socialisation en dehors des entreprises.

Si la création de liens entre les entreprises actuellement présentes dans le bâtiment semble difficile, tel n'a pas toujours été le cas et l'édifice RCA semble avoir été, pour reprendre les mots sans équivoque d'un de nos répondants, le siège "d'une communauté artistique dynamique et créative [qui s'est peu à peu transformée] en une collection somnolente et engourdie de nerds informatiques autocentrés ». Cette communauté artistique comptait en effet plusieurs dizaines d'artistes dans les années 2000 . Il en reste aujourd'hui une douzaine. Les interactions entre ces artistes semblent avoir été nombreuses et les liens qui les unissaient assez forts. De plus, cette communauté perdue contribuait au rayonnement du bâtiment dans le quartier et au-delà, notamment en organisant régulièrement des événements culturels.

Une communauté patrimoniale? La valeur symbolique attachée au bâtiment et à son histoire pourrait devenir une ressource pour les entreprises du RCA, qui auraient al ors intérêt à la préserver et à la mettre en avant, dynamique qui pourrait constituer les bases d'une communauté patrimoniale ${ }^{37}$.

En consultant les sites Internet des entreprises du RCA, nous n'en avons repéré que douze qui font référence à leur localisation dans un immeuble patrimonial. Le passé industriel du bâtiment est également valorisé dans la décoration 


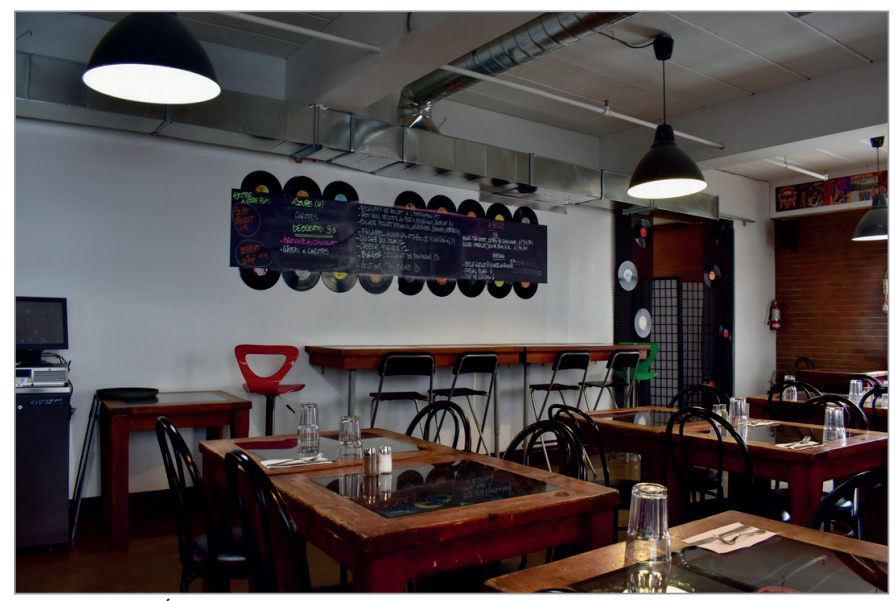

ILL. 14. LE CAFÉ ZINZIN. | LAURENT DAMBRE-SAUVAGE, 9 AVRIL 2018.

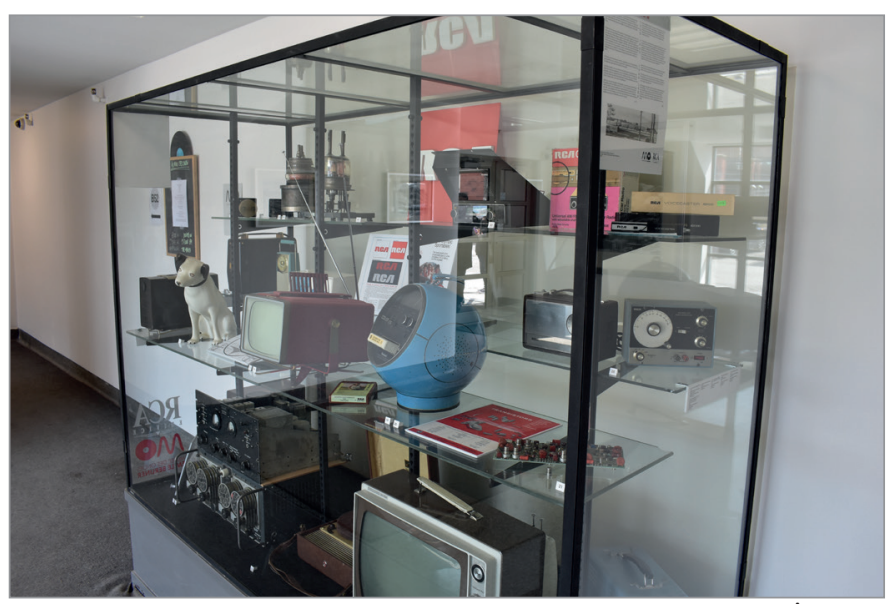

ILL. 15. VITRINE MISE EN PLACE PAR LE MOEB DANS LES ESPACES COMMUNS DU BÂTIMENT. | LAURENT DAMBRE-SAUVAGE, 9 AVRIL 2018 du Café Zinzin, qui est constituée de disques vinyles ornant les murs et les tables (ill. 14). Il faut cependant relever le faible nombre d'entreprises puisant à la ressource patrimoniale du lieu.

Dans le bâtiment, le MOEB œuvre à titre de "passeur de mémoire " et promeut ses valeurs d'existence (principalement sa valeur d'âge - en liant le bâtiment à I'histoire industrielle du Canada - et sa valeur de matérialité - en mettant en avant ses caractéristiques techniques). Ce faisant, il alimente le capital symbolique lié au RCA, capital qui pourrait être utilisé comme ressource par les divers acteurs de l'écosystème. La promotion de ces valeurs d'existence par le MOEB se fait tant en interne (vers les gens qui utilisent régulièrement le bâtiment), notamment par la mise en place, dans les espaces communs, de vitrines présentant des objets de le collection (ill. 15), qu'en externe (les visiteurs, les touristes).

Les propriétaires mettent également en avant le caractère patrimonial du lieu sur leur site Internet ${ }^{38}$ et assurent une aide au MOEB, en proposant des espaces d'exposition et d'entreposage à prix avantageux ou en leur permettant de disposer des vitrines d'exposition dans les couloirs.

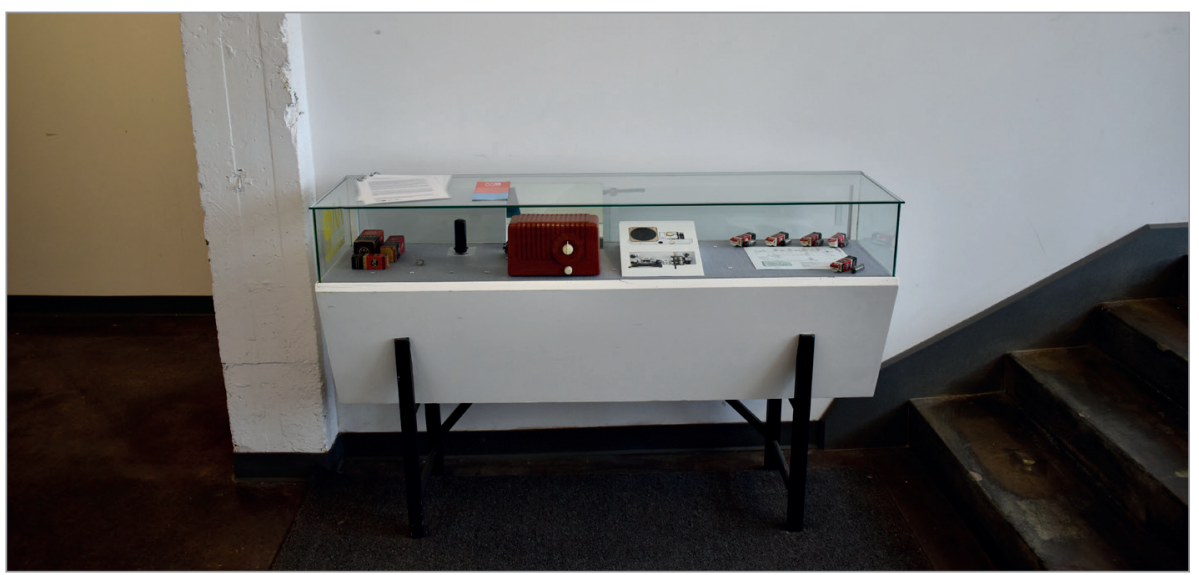

ILL. 16. VITRINE MISE EN PLACE PAR LE MOEB DANS LES ESPACES COMMUNS DU BÂTIMENT. | LAURENT DAMBRE-SAUVAGE, 9 AVRIL 2018.

L'alliance entre le MOEB et les propriétaires pourrait constituer l'embryon d'une communauté patrimoniale, celle-ci peine cependant à émerger. L'histoire du bâtiment reste en effet peu appropriée par les usagers qui mettent en avant plutôt la fonctionnalité des espaces et le côté "cool» de l'ambiance industrielle des lofts. Les personnes que nous avons interrogées connaissent plus ou moins vaguement I'histoire du bâtiment et le MOEB suscite peu d'intérêt.

La faiblesse des moyens du MOEB peut expliquer cet état de fait, en limitant ses possibilités de rayonnement. L'espace d'exposition est très petit et la muséographie des vitrines peu engageante (ill. 16). De plus, la logique du modèle économique des propriétaires les invite à limiter la philanthropie : les espaces attribués au musée se réduisent et le musée est amené, pour cette raison parmi d'autres, à se départir de certaines pièces de ses collections.

\section{Une faible valeur de reconnaissance}

Malgré le travail de certains acteurs pour promouvoir et préserver l'édifice RCA, la reconnaissance de la valeur historique et 

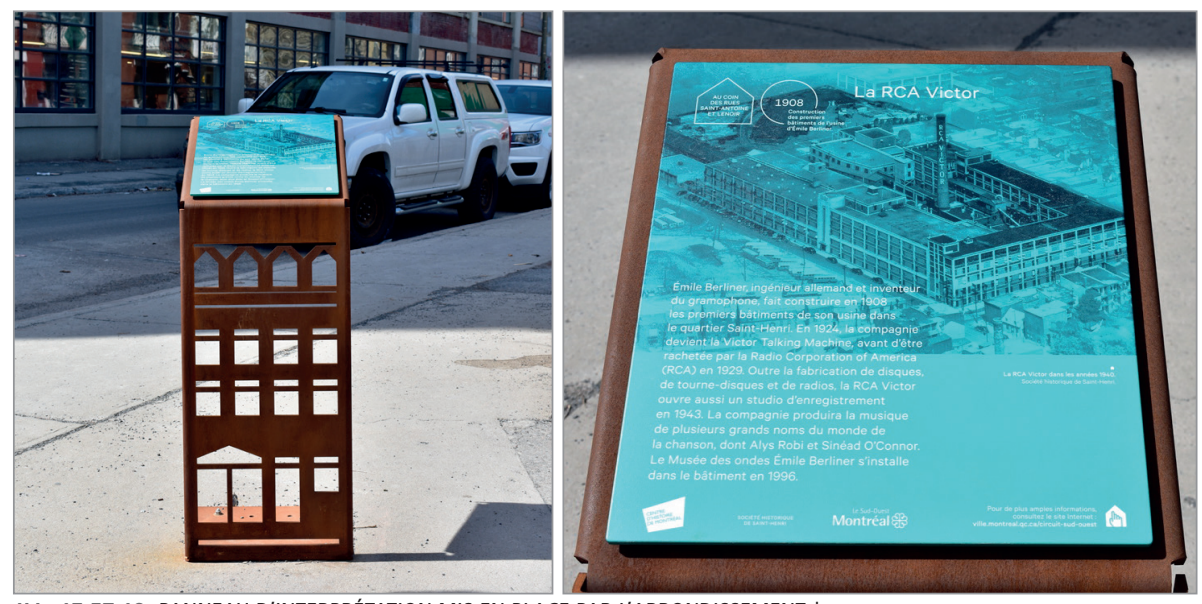

ILL. 17 ET 18. PANNEAU D'INTERPRÉTATION MIS EN PLACE PAR L'ARRONDISSEMENT. | LAURENT DAMBRE-SAUVAGE, 9 AVRIL 2018.

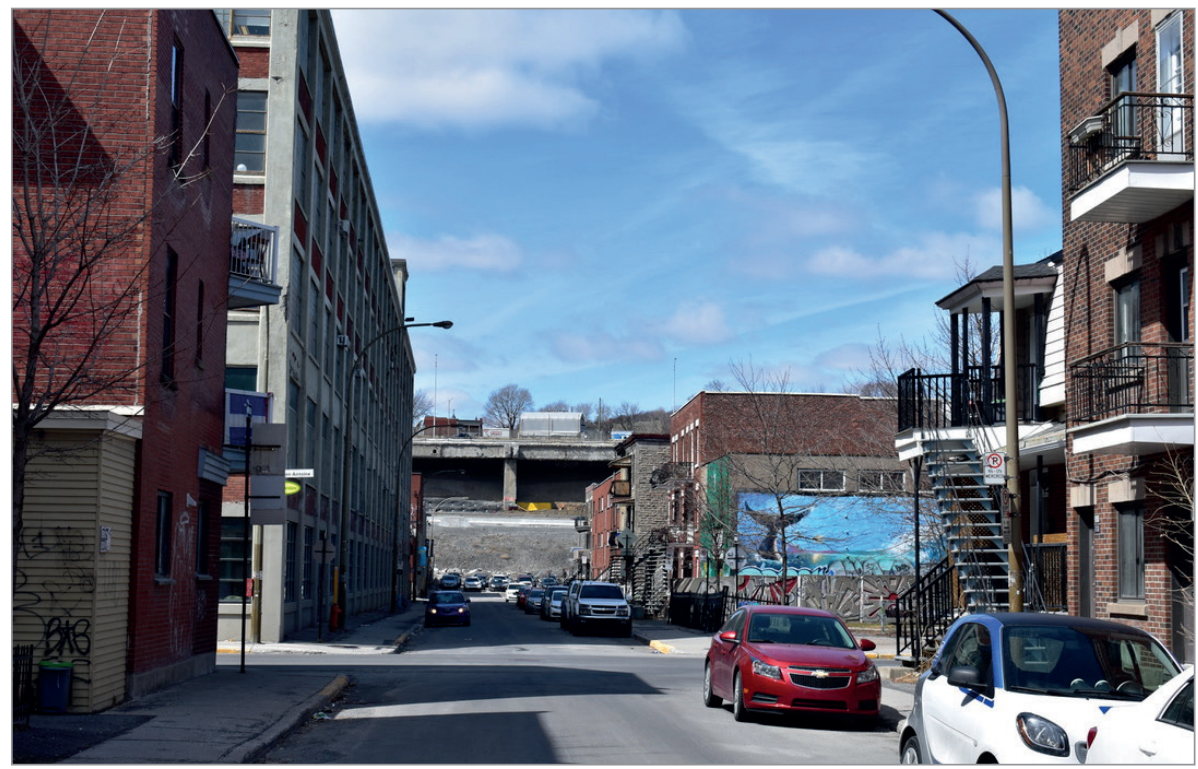

ILL. 19. LE BÂTIMENT DANS SON CONTEXTE URBAIN, EN CONTREBAS DE L'AUTOROUTE VILLE-MARIE. | LAURENT DAMBRE-SAUVAGE, 9 AVRIL 2018.

patrimoniale du lieu à l'intérieur de son propre écosystème est plutôt faible, ce qui invalide l'émergence d'une communauté patrimoniale. À cette faible valeur de reconnaissance interne correspond une faible valeur de reconnaissance externe.

Si l'immeuble est identifié comme un immeuble d'intérêt patrimonial dans le Plan d'urbanisme de Montréal, son absence de classement est perçue par les acteurs rencontrés comme une absence de reconnaissance par les pouvoirs publics, absence que des répondants soulignent en notant que "l'immeuble n'est pas un immeuble patrimonial».

De plus, malgré l'implication d'habitants du quartier et d'anciens travailleurs de I'usine dans le musée, la reconnaissance de la valeur du bâtiment par les habitants semble faible. Les habitants que nous avons interrogés, pourtant membres de la Société historique de Saint-Henri, ont souligné le manque d'intérêt architectural de l'immeuble, sa «laideur» même parfois.

Il faut cependant tempérer ce manque de reconnaissance en relevant le fait que la mairie d'arrondissement, en collaboration avec la Société historique de Saint-Henri, met en valeur le RCA en l'incluant dans des circuits patrimoniaux ou en installant des panneaux d'interprétation renseignant le promeneur sur son histoire (ill. 17 et 18 ).

Selon nous, la faiblesse de la valeur de reconnaissance du RCA doit être mise en relation avec le fait que sa valeur de position pose problème. Le bâtiment est situé dans un cul-de-sac, en contrebas de I'autoroute Ville-Marie (ill. 19), dans une partie de Saint-Henri qui porte des marques visibles de « défavorisation " (certains bâtiments aux alentours sont plutôt délabrés). Peu de commerces sont situés aux alentours, en dehors d'une grande surface de bricolage et de son parking qui concourent à l'enlaidissement des abords du bâtiment. Par ailleurs, I'environnement proche est également dégradé par la présence de terrains vagues qui servent d'aires de stationnement. Enfin, la configuration même d'un bâtiment qui apparaît comme fermé sur l'extérieur participe à l'isoler des dynamiques urbaines dans lesquelles il s'insère.

L'ensemble de ces facteurs fait en sorte que les habitants du quartier ne fréquentent ni le bâtiment ni ses alentours. Dans ces conditions, le bâtiment se situe dans une espèce d'angle mort qui rend difficile toute reconnaissance de la part des habitants du quartier ou des visiteurs.

À l'issue de cet examen des différentes valeurs portées par le projet, on peut résumer les forces et les faiblesses du 
modèle développé ici. D'un côté, il est autosuffisant et permet d'assurer la préservation et la restauration du bâtiment dont il développe et consolide l'usage. II contribue également de manière positive au développement économique du quartier en y créant de l'emploi. D'un autre côté, les valeurs communautaires induites par ce projet sont faibles, notamment en ce qui concerne les locataires les plus récents qui sont quelque peu isolés au sein de l'écosystème RCA quand d'autres communautés, plus anciennes, se sont, peu à peu, décomposées. Les valeurs symboliques liées à la dimension patrimoniale du bâtiment et aux représentations qu'il suscite ne sont pas investies par la majorité des entreprises et des artistes locataires. Enfin, il faut également souligner la faible valeur de reconnaissance tant par les usagers du bâtiment, les habitants du quartier et les acteurs publics.

Si le bâtiment n'est actuellement pas en danger, on peut s'interroger sur son avenir à long terme, notamment en cas de changement de propriétaire. Dès lors, nous souhaitons proposer un plan d'action qui permettrait la préservation à long terme du bâtiment et la maximisation de ses effets sur le développement local.

\section{PHASE PROSPECTIVE :} PROPOSITION D'UN PLAN D'ACTION

En partant des constats faits plus haut, nous voulons ici faire un exercice d'imagination et proposer des solutions pour consolider le devenir du bâtiment en maximisant son impact dans le développement socioéconomique du quartier.

Constatant l'absence de péril pesant de manière immédiate sur le bâtiment, ce plan d'action a pour but ultime de favoriser l'émergence d'une communauté suffisamment dynamique pour élaborer des solutions alternatives fortes et crédibles à la reprise du bâtiment par un propriétaire privé en cas de vente. Nous partons du présupposé que le propriétaire actuel est convaincu que favoriser l'émergence d'un cluster d'entreprises peut avoir un impact positif sur la valeur locative de ses espaces et freiner le roulement des locataires, ce qui constitue une motivation l'amenant à s'engager dans une démarche qui vise à renforcer à la fois les relations entre les membres des communautés articulées autour du bâtiment et l'attachement de ces communautés à l'édifice en lui-même pour en faire un élément structurant d'une identité collective.

Ce plan pourrait se décliner en trois objectifs.

\section{1) Favoriser l'émergence d'une communauté entrepreneuriale}

Partant du constat que la coprésence, dans un même lieu, d'entreprises qui pourraient articuler des relations autour d'une même chaîne de production de valeur et développer des liens leur permettant de favoriser les processus d'innovation, il s'agit de favoriser l'émergence d'un véritable cluster d'entreprises créatives. Celui-ci pourrait à la fois constituer une source de plus-value pour les entreprises et un facteur de désirabilité pour le bâtiment.

Préserver la diversité des entreprises présentes au sein du RCA. Cette diversité concourt à la complémentarité des entreprises présentes dans le RCA tout en permettant de croiser les savoirs et les expertises, ce qui peut provoquer l'émergence d'innovations. En conduisant à l'éviction de certains acteurs plus faibles économiquement (artistes, jeunes entreprises), le modèle actuel peut nuire à cette diversité. Nous proposons la mise en place, par les propriétaires, d'une politique de fixation des loyers qui préserve les acteurs les moins forts économiquement (création d'espaces plus petits mais bien conçus pour leurs activités, augmentation raisonnable des loyers pour les acteurs les plus importants), ce qui revient à s'engager dans une logique d'entrepreneuriat social (au sens américain du terme), où la génération de revenus d'une activité marchande alimente une mission sociale (qui ici peut être double : préservation du patrimoine et aide aux entreprises ou aux artistes).

Favoriser l'installation et la visibilité d'entreprises permettant les rencontres entre acteurs. Il y a dans le bâtiment un certain nombre d'entreprises qui peuvent constituer des lieux de rencontres et d'échanges pour les personnes qui travaillent dans le bâtiment (garderie, cafés, restaurants, gym). Toutefois, ceux-ci sont peu nombreux et peu visibles. Les propriétaires auraient intérêt à maintenir la présence de la garderie et du gymnase dans les étages sur rue, et à promouvoir l'installation de cafés et de restaurants sur ces mêmes étages.

Par ailleurs, il serait intéressant de développer des espaces communs non marchands, par exemple en utilisant les cours et les toits pour aménager des endroits mis à la disposition des employés (fumoirs, aires de repas, aires ludiques), constituant des lieux de rencontres et d'échanges informels.

Améliorer la communication en interne entre les acteurs du bâtiment. Les entretiens ont révélé que les entreprises abritées dans le bâtiment ne se connaissaient pas entre elles. Pour rompre cette méconnaissance, nous proposons la création d'une plateforme informatique qui renfermerait, entre autres, un répertoire des entreprises, constituerait un outil 
de communication interne et pourrait faire circuler des offres d'emplois ou de services.

Engager un animateur de communauté (community manager) pour faire vivre la communauté. Les actions énoncées dans cet objectif requièrent la mobilisation d'une ressource à temps plein. Nous proposons donc l'engagement par les propriétaires d'une personne dédiée à l'animation communautaire du lieu.

\section{2) Développer la valeur}

de reconnaissance du bâtiment

et de son histoire

Le bâtiment, on l'a vu, souffre d'un manque de reconnaissance qui s'incarne à la fois dans des statuts administratifs vécus de manière négative par les acteurs et par une vision plutôt dégradée des habitants du quartier. Travailler à développer la valeur de reconnaissance du bâtiment pourrait, selon nous, constituer un projet rassembleur susceptible de faire émerger une communauté patrimoniale fière de son histoire. Ce travail pourrait être porté par une coalition rassemblant les propriétaires, le MOEB, la mairie d'arrondissement, auxquels viendraient s'agréger peu à peu d'autres acteurs (locataires, habitants). Des financements pourraient être sollicités auprès des financeurs culturels classiques (Conseil des arts de Montréal, Conseil des arts et des lettres du Québec, Conseil des arts du Canada).

Pour ce faire, nous proposons une série d'actions destinées, à l'interne, aux acteurs de l'écosystème (les deux premières) et, à l'externe, aux visiteurs potentiels (les troisième et quatrième) :

Améliorer les vitrines sur l'histoire du bâtiment. Les vitrines mises en place dans les couloirs du bâtiment par le MOEB présentent une qualité muséographique médiocre, ne répondant pas aux attentes de personnes qui évoluent dans le milieu créatif. Nous proposons donc de mener une réflexion pour mettre en place une muséographie plus satisfaisante pouvant, le cas échéant, faire appel aux technologies de l'information.

Utiliser les compétences des personnes travaillant dans les locaux pour la mise en valeur du bâtiment. Les locataires du RCA représentent un stock important de savoir-faire (artistique, technologique, administratif...) qui pourrait être mis au service du projet patrimonial. L'activation de ces savoir-faire pourrait, en outre, constituer un moyen d'émulation et renforcer les liens entre les entreprises. Nous proposons donc de solliciter les locataires, par le bais de concours ou la mise en place d'incitatifs financiers, pour développer des projets mettant en valeur le bâtiment et son histoire (murales, réalité augmentée...).

Assurer une meilleure visibilité des passeurs de mémoire. L'emplacement du MOEB au cœur du bâtiment nuit à sa visibilité. Nous suggérerions donc aux propriétaires d'installer le musée dans des locaux visibles et accessibles depuis la rue. De plus, le MOEB suscite l'implication dans ses activités d'une communauté d'anciens travailleurs de la société RCAVictor. Ceux-ci sont les dépositaires d'une histoire qu'il conviendrait de mettre en valeur, notamment en recueillant et diffusant leur témoignage.

Mettre en valeur I'histoire du bâtiment par des activités culturelles. L'histoire du bâtiment est fortement liée à I'histoire musicale du Québec. La mise en place d'activités culturelles pourrait contribuer à faire connaître cette histoire aux habitants du quartier et aux visiteurs. Un partenariat avec la Hacienda Creative pourrait permettre de développer, dans le studio, une activité événementielle récurrente. De larges espaces à I'arrière du bâtiment constituent des lieux intéressants pour organiser un festival musical d'été. Enfin, la présence d'artistes et de galeries pourrait également être l'occasion d'organiser des événements culturels de type portes ouvertes. Ces actions pourraient s'inscrire dans un grand plan de mise en valeur de l'histoire musicale du Sud-Ouest et, plus particulièrement, de l'importance du quartier dans I'histoire du jazz.

Consacrer cette dynamique dans I'élaboration de dossiers de demande de reconnaissance (Québec, Canada). On pourrait s'inspirer de l'exemple d'Arvida qui montre les effets positifs sur le tissu social d'un territoire de l'élaboration de demandes de reconnaissance d'un lieu via son inscription dans les inventaires du patrimoine (à quel que niveau que ce soit). Cette démarche, si elle est soutenue par les populations locales, contribue à renforcer les liens communautaires et les sentiments de fierté et d'appartenance. Nous proposons donc la constitution de demandes d'inscription à l'inventaire du patrimoine, aux niveaux provincial et fédéral, au titre de la valeur exceptionnelle du bâtiment quant au rôle d'Emile Berliner, à l'importance du bâtiment dans I'histoire technique, aux qualités techniques et architecturales du bâtiment et à son rôle de jalon dans I'histoire musicale.

\section{3) Assurer une meilleure intégration de l'immeuble dans l'environnement urbain, consacrant sa place moteur dans le développement social et économique du quartier}

Il s'agit ici de briser le côté fermé du bâtiment pour faciliter la pénétration des dynamiques urbaines du quartier dans lequel il s'insère. Pour atteindre ce 
troisième objectif, nous proposons trois séries d'actions :

Favoriser, au rez-de-chaussée de l'immeuble, l'installation d'entreprises pouvant desservir aussi bien les besoins des locataires que ceux des habitants. Cela pourra permettre au bâtiment de devenir une destination pour les habitants du quartier. Plus particulièrement, il s'agira de combler un certain nombre de besoins commerciaux non satisfaits dans le quartier.

Élaborer un design permettant de rompre le côté fermé de l'immeuble sur la rue tout en préservant ses spécificités architecturales.

Améliorer l'environnement urbain, notamment dans les parties délaissées à l'arrière du bâtiment.

Cela pourrait se faire par la mise en place d'un Plan particulier d'urbanisme destiné à revitaliser un secteur de Saint-Henri qui témoigne d'un certain nombre de marqueurs de problématiques socioéconomiques (habitat insalubre, environnement dégradé...).

En conclusion, le modèle de projet patrimonial présenté dans le cas du bâtiment RCA, reposant sur l'activation de dynamiques capitalistes mises en place par des acteurs privés, mettant au service de la préservation du bâtiment des profits générés par l'usage de ce dernier, présente un certain nombre d'avantages, dont la préservation du bâtiment et le non-recours aux subventions. En outre, il apporte une contribution non négligeable au développement économique du quartier, en y créant de l'emploi. Cependant, ce modèle comporte des limites en ce qui concerne le développement social du quartier dans la mesure où il génère peu de valeurs dynamiques.
Par ailleurs, la logique de maximisation des profits conduit à l'exclusion de certains locataires et préside à des choix qui restreignent l'action d'un passeur de mémoire important : le MOEB. De plus, la stabilité de ce modèle dans le temps doit être remise en question. Enfin, l'engagement des propriétaires dans une démarche d'entrepreneuriat social affirmée où les ressources marchandes générées par la location d'espaces seraient mises au service du développement d'une communauté autant qu'à la préservation du patrimoine pourrait compenser, en partie, les faiblesses du projet.

\section{NOTES}

1. Les réflexions présentées ici sont issues du séminaire "Patrimoine et développement local » donné à l'hiver 2018 à l'Université du Québec à Montréal, par Lucie K. Morisset, que nous tenons ici à remercier très chaleureusement : non seulement ce travail lui doit beaucoup, mais les échanges entamés à cette occasion continuent à inspirer mes recherches sur les communs culturels.

2. Morisset, Lucie K., 2009. Des régimes d'authenticité. Essai sur la mémoire patrimoniale Québec, Presses de I'Université du Québec, Rennes, Presses universitaires de Rennes.

3. Moogk, Edward B., 1975, En remontant les années: I'histoire et l'héritage de l'enregistrement sonore au Canada : des débuts à 1930, Ottawa, Bibliothèque nationale du Canada.

4. Leduc, Louise, 1997, « La grande petite histoire du musée Berliner », Le Devoir, 13 décembre, p. B3.

5. Laurence, Jean-Christophe, 2010, "Un chien célèbre ", La Presse, 6 février, p. 7.

6. Laurence, Jean-Christophe, 2010, "Journées de la culture ", La Presse, 24 septembre, p. 2.

7. Rakobowchuk, Peter, 2017, "Museum Highlights Gramophone Inventor », Hamilton Spectator, 16 septembre, p. G13.

8. Anonyme, 2015, «Exposition sur la radio montréalaise en temps de guerre ", La Voix Pop, 7 mai, p. 14.

9. Brunet, Alain, 2015, "Le Studio Victor ferme ses portes ", La Tribune, 3 décembre, p. 21.
10. Desroches, André, 2015. "Le Studio Victor ferme ses portes après 30 ans ", La Voix Pop, 7 décembre, p. 5 .

11. Lyan, Marie, 2018, "La Hacienda Creative s'installe au studio Victor ", Les Affaires, 11 janvier, [https://www.lesaffaires.com/strategie-d-entreprise/pme/la-hacienda-creatives-installe-au-studio-victor/599498], consulté le 16 février 2018.

12. Leduc, "La grande petite histoire du musée Berliner », op. cit., p. B3.

13. Abastaldo, Marie-France, 2016, La grande histoire du son et de l'enregistrement à Montréal au XXe siècle, 12 août, [https://ici. radio-canada.ca/nouvelle/764965/museeondes-emile-berliner-radio-histoire-rca-victortelevision-appareil-ondes-visite], consulté le 16 février 2018.

14. Rakobowchuk, Peter, 2017, "The History of Sound ", The Guardian, Charlottetown, 22 septembre, p. C10.

15. Anonyme, 2007, "Three Local Talents Highlight ART@RCA Open Studio Event », The Westmount Examiner, 10 mai, p. 10.

16. Joncas, Hugo, 2008, "Le Centre RCA de Saint-Henri sera restauré ", Les Affaires, 29 novembre, p. 22.

17. Éric, Clément, 2009, "Tentatives d'extorsion enregistrées ", La Presse, 9 janvier, p. 6.

18. Ici Radio-Canada, 2015, «Montréal : le fameux studio Victor fermera ses portes à la fin du mois ", Le Téléjournal, 19 décembre.

19. Desroches, "Le Studio Victor ferme ses portes après 30 ans », op. cit., p. 5.

20. Ici Radio-Canada, "Montréal : le fameux studio Victor fermera ses portes à la fin du mois ", op. cit.

21. Lyan, « La Hacienda Creative s'installe au studio Victor ", op. cit.

22. [http://www.moeb.ca/notre-musee-desondes], consulté le 15 février 2018.

23. Ibid.

24. Depuis la rédaction de cet article, le bâtiment est devenu la propriété du groupe Allied Properties REIT.

25. Joncas, "Le Centre RCA de Saint-Henri sera restauré ", op. cit., p. 22.

26. [http://gestioncoulombe.com/georges-coulombe-gestionnaire-immobilier/], consulté le 15 février 2018. 
27. Joncas, " Le Centre RCA de Saint-Henri sera restauré », op. cit., p. 22.

28. Le propriétaire annonce, quant à lui, le nombre de 240

29. [http://kollectif.net/projet-du-mois/49475/, consulté le 15 février 2015

30. Id.

31. Ville de Montréal, 2005, Évaluation du patrimoine urbain. Arrondissement du SudOuest, Montréal, p. 55.

32. Joncas, " Le Centre RCA de Saint-Henri sera restauré ", op. cit., p. 22.

33. Klein, Juan-Luis et Diane-GabrielleTremblay (dir.), 2020, Les indicateurs de vitalité culturelle dans l'arrondissement du Sud-Ouest (Montréal), Cahiers du Crises (Centre de recherche sur les innovations sociales), Montréal, [https://crises.uqam.ca/wp-content/ uploads/2020/03/CRISES ET2002.pdf], consulté le 15 septembre 2020.

34. Sur les communs dans le secteur de la culture et du patrimoine, voir, par exemple, Bertacchini, Enrico, Giangiacomo Bravo, Massimo Marrelli et Walter Santagata, 2012, Cultural Commons: A New Perspective on the Production and Evolution of Cultures, Northampton, Edward Elgar Publishing.
35. Scott, Allen J. et Frédéric Leriche, 2005, « Les ressorts géographiques de l'économie culturelle : du local au mondial ", L'Espace géographique, vol. 34, n³, p. 207-222; Sacco, Pier Luigi, Giorgio Tavano Blessi et Massimiliano Nuccio, 2013, "Culture as an Engine of Local Development Processes: System-wide Cultural Districts I: Theory ", Growth and Change, vol. 44, n 4, p. 555-570.

36. Scaillerez, Arnaud et Diane-Gabrielle Tremblay, 2017, " Coworking, Fab Labs et Living Labs », Territoire en mouvement Revue de géographie et aménagement, 34 I 2017, mis en ligne le 16 juin, [http://tem.revues. org/4200], consulté le 6 juillet 2017.

37. Conseil de I'Europe, 2005, Convention-cadre du Conseil de l'Europe sur la valeur du patrimoine culturel pour la société, Série des traités du Conseil de l'Europe, n 199, 27 octobre 2005.

38. [http://www.edificerca.com/fr/historique/], consulté le 15 février 2018 (page supprimée depuis). 